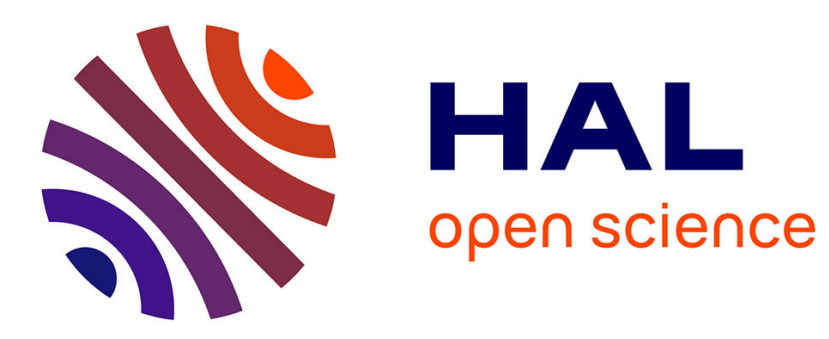

\title{
Signal detection using watermark insertion
}

Matthieu Gautier, Dominique Noguet

\section{To cite this version:}

Matthieu Gautier, Dominique Noguet. Signal detection using watermark insertion. IEEE International Vehicular Technology conference (VTC-Spring13), Jun 2013, Dresden, France. pp.11. hal00833552

\section{HAL Id: hal-00833552 \\ https://hal.science/hal-00833552}

Submitted on 13 Jun 2013

HAL is a multi-disciplinary open access archive for the deposit and dissemination of scientific research documents, whether they are published or not. The documents may come from teaching and research institutions in France or abroad, or from public or private research centers.
L'archive ouverte pluridisciplinaire HAL, est destinée au dépôt et à la diffusion de documents scientifiques de niveau recherche, publiés ou non, émanant des établissements d'enseignement et de recherche français ou étrangers, des laboratoires publics ou privés. 


\title{
Signal detection using watermark insertion
}

\author{
Matthieu Gautier ${ }^{1}$ and Dominique Noguet ${ }^{2}$ \\ ${ }^{1}$ IRISA, INRIA, University of Rennes, France \\ ${ }^{2}$ CEA-LETI, Minatec, Grenoble, France \\ matthieu.gautier@irisa.fr, dominique.noguet@cea.fr
}

\begin{abstract}
This paper analyses signal detection using watermark insertion, which is artificially embedded into the digital modulated signal. When a signal does not contain intrinsic information, its detection is hard to achieve using blind detectors. For that kind of signals, we propose to insert a low-power watermark that will be detected by a matched filter based detector. The system design is a trade-off between the watermark insertion strength (i.e. reducing the transmission quality) and the detection sensitivity. This trade-off is discussed in this paper and simulations results show the advantage of the watermark insertion.
\end{abstract}

\section{INTRODUCTION}

Cognitive radio systems [1] can use different approaches to determine the spectrum occupancy. The first is to query a database of the positions of the radio transmitters and their parameters (power, frequency) in order to deduce a map of the spectrum occupation. Another approach is to directly detect the signals using spectrum sensing techniques. If the first can protect perfectly the incumbents, it cannot help the management of resource allocation among opportunistic users. Detection techniques must be specifically adapted to the opportunistic users. The detection of opportunistic users may be considered by the joint approach of the design of the physical layer of opportunistic users and the design of their detection.

In the literature, many detection techniques have been proposed [2]. Those that offer the highest levels of sensitivity for a given detection time are those that use a priori information of the signal. However, some signals do not contain many intrinsic signatures, in that case only blind algorithms can detect them, such as the energy detector [3].

The solution proposed in this paper relies on the following: the detection can be assisted by the explicit introduction of a specific signature (or watermark) in the transmitted signal.

The introduction of signatures in the transmitted signal has already been addressed in the literature [4][5]: they propose to insert cyclostationary signatures on some carriers of the signal to obtain an efficient detection of the signal. These solutions are highly dependent on the modulation used (OFDM [6], FilterBank MultiCarrier (FBMC) [7], ...) and reduce the spectral efficiency of the system. Unlike [4][5], the proposed scheme is to insert a signature independent of the modulation used. It does not insert cyclostationary features into the signal but adds a watermark [8][9]. The detection scheme differs from conventional detectors because it relies on the detection of the watermark, not the detection of the useful signal.

In our approach, the watermark is inserted with a very low power not to alter the signal significantly. Thus, a receiver must be able to demodulate the signal without any knowledge on the watermark. Only the detector uses the watermark to detect the signal.

This paper consists of 5 parts. Section II describes the system model and introduces the watermark based detector. In Section III, a theoretical analysis of the detector is introduced in order to set the system. Simulation results are given in Section IV. Finally, conclusions are drawn and outlook is provided.

\section{SYSTEM MODEL}

In order to introduce the system, we consider the study case where an opportunistic user "User 2" wants to use the spectrum white spaces of incumbent systems. Before performing its communication, this user want to detect whether or not the free bands are already occupied by another opportunistic user "User 1". The principle of the proposed detection is to insert a watermark in the transmitted signal and to use this watermark to help the detection of User 1 by other opportunistic users. Fig. 1 describes the system model of the watermark insertion in the signal of User 1 and its detection by User 2. Two communication paths are described:

- the transmission of User 1 through Channel 1 in order to test the influence of the watermark insertion on the quality of the User 1 demodulation,

- the transmission of User 1 through Channel 2 in order to test the quality of the detection of User 1 by User 2 .

Under specific circumstances where User 2 wants to detect and demodulate the modulated signal, Channel 1 and Channel 2 are identical.

\section{A. Watermarking the transmitted signal}

The proposed watermarking scheme is inspired from the watermarking of audio signals described in [9] where the hidden information is embedded inside into an audio signal. The watermarking consists in adding the signal $w(t)$ with

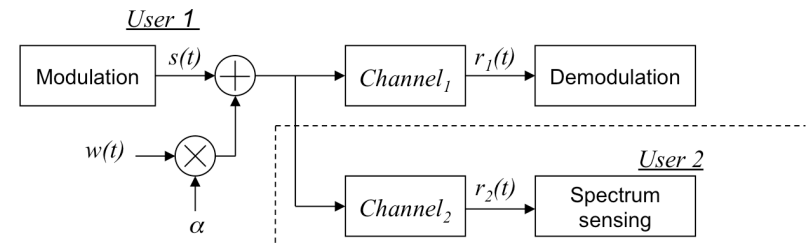

Fig. 1. System model of the watermark insertion in the signal of User 1 and its detection by User 2 . 


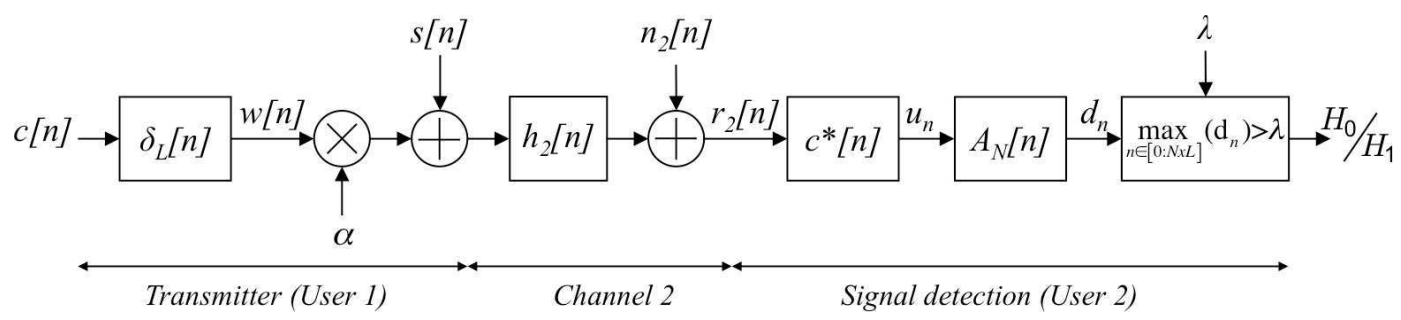

Fig. 2. Implementation of the watermark insertion and detection.

the signal $s(t)$ as shown in Fig 1. The watermark power being normalized $\sigma_{w}^{2}=1$, the power of the watermark insertion in the signal $s(t)$ depends only on the coefficient $\alpha$ and is characterized by the ratio WSR (Watermark to Signal Ratio) given by:

$$
W S R=10 . \log _{10}\left(\frac{\alpha^{2}}{\sigma_{s}^{2}}\right),
$$

with $\sigma_{s}^{2}$ the power of the transmitted signal.

Considering the transmission between the modulation and the demodulation of User 1, the Signal-to-Noise Ratio $(S N R)$ in an AWGN (Additive White Gaussian Noise) channel characterizes the transmission quality by:

$$
S N R=10 \cdot \log _{10}\left(\frac{\sigma_{s}^{2}}{\sigma_{n}^{2}+\alpha^{2}}\right),
$$

with $\sigma_{n}^{2}$ the noise power.

\section{B. Watermark detection}

The watermark inserted into User 1 should improve its detection by other users. The aim of the detector is to detect the presence of the watermark, not to detect the signal itself. Thus, the detection can be stated as the following hypothesis (in the case of an AWGN noise $n_{2}(t)$ ):

$$
\left\{\begin{array}{l}
H_{0}: r_{2}(t)=n_{2}(t) \\
H_{1}: r_{2}(t)=s(t)+\alpha w(t)+n_{2}(t)
\end{array} .\right.
$$

where $H_{0}$ is the null hypothesis for the event "free band" and $H_{1}$ is the alternative hypothesis for the event "occupied band". The detection is performed by the matched filter to the watermark. The correlation between the received signal and the watermark is computed so that the receiver must known the watermark. The output of the correlation $u$ is given by:

$$
u=\frac{<r_{2}, w^{*}>}{N_{s}}= \begin{cases}\frac{\left\langle n_{2}, w^{*}>\right.}{N_{s}} & \text { if } H_{0}, \\ \frac{\left\langle s+n_{2}, w^{*}>\right.}{N_{s}}+\alpha & \text { if } H_{1} .\end{cases}
$$

$N_{s}$ is the detection duration i.e. the number of samples used to compute the correlation.

Finally, the decision variable $T$ is computed:

$$
T=\max (E[u]),
$$

where $E[u]$ is the mathematical expectation operator.

\section{Implementation issues}

In a practical implementation, the watermark will be inserted in the baseband signal just before the digital to analog conversion (DAC). Fig. 2 shows the digital model used to test our detection scheme. At the transmitter side, a pattern code $c(t)$ is repeated to generate the infinite-duration signal $w(t)$. The digital expression of $w(t)$ is:

$$
w[n]=c * \delta_{L}[n] \text { with } \delta_{L}[n]=\sum_{k=-\infty}^{+\infty} \delta[n-k L],
$$

with $L$ the number of samples of the code $c[n]$ and, $\delta$ and $\delta_{L}$ the Dirac and the Dirac comb functions respectively.

The choice of the code is important. It is characterized by its length $L$ and must have good auto-correlation/crosscorrelation properties to be easily detectable. It must also be uncorrelated with the useful signal.

In our simulations, we first consider that the watermark is a Gaussian random sequence i.e., $w(t) \sim \mathcal{N}\left(0, \sigma_{s}^{2}\right)$. This leads to ideal autocorrelation property. Then, we used the codes of literature conventionally used in spread spectrum systems [10] as the UMTS i.e. the Hadamard codes. These codes are orthogonal, however this feature is not essential and, we could also use pseudo-random codes, non-orthogonal, such as the Gold codes.

To set the system, the most important parameter is the insertion power $\alpha$ characterized by the ratio $W S R$ defined in (1). This ratio should be as low as possible so that the insertion has the least influence on the useful transmitted signal. Typically, the simulation results show that a ratio of $-15 \mathrm{~dB}$ does not degrade system performance (see Section 3).

At the signal detection side in Fig. 2, the correlation between the received signal and the code used at the transmitter side is performed. Then the expectation operation in (5) is estimated. The correlation $u[n]$ is computed using the matched filter of the code:

$$
u[n]=r_{2} * c^{*}[n] .
$$

The expectation operation is performed by a first order IIR (Infinite Impulse Response) filter $A_{N}$ :

$$
A_{N}[n]=\sum_{k=0}^{N} \delta[n-k L] .
$$




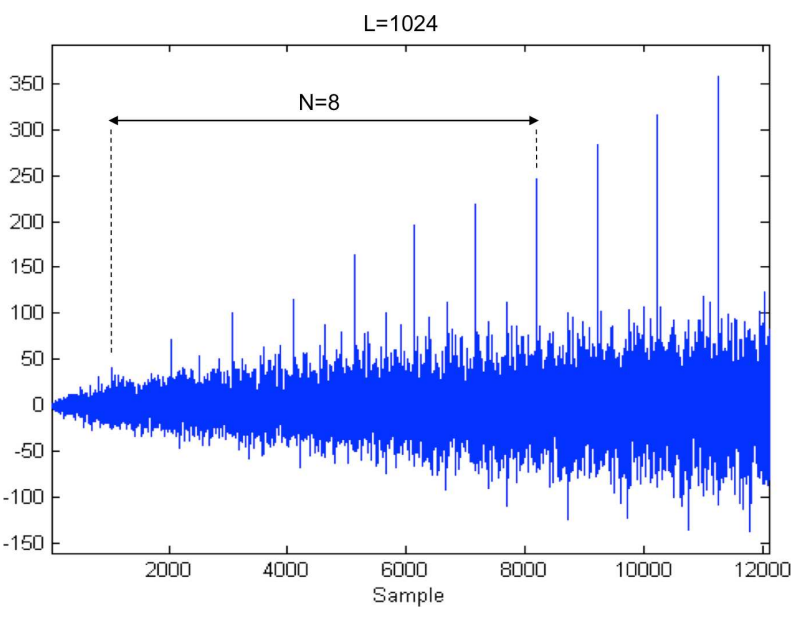

Fig. 3. Output of the averaging filter $d_{n}$.

The length of the filter is $N$ and is directly responsible of the signal duration needed to provide the decision.

$L$ is the number of taps of the matched filter, the number of samples $N_{s}$ used for the detection is:

$$
N_{s}=N * L \text {. }
$$

By increasing $N$, the accuracy of the estimation is improved and therefore the algorithm can detect the presence of the watermark with a lower $S N R$. Fig. 3 illustrates how the detector operates by showing the output of the averaging filter. It shows the correlation peaks that increase gradually as the average is calculated.

Once the average performed, we come to the decision by comparing the maximum output of the filter to a decision threshold $\lambda$.

\section{THEORETICAL PERFORMANCE}

This section presents the theoretical performance of the proposed system and a validation with simulation results. These results are important to set the system's parameters.

\section{A. Watermark insertion sensibility}

First, the influence of the watermark on the transmission quality of User 1 must be evaluated. Taking Gaussian assumptions $\left(s(t) \sim \mathcal{N}\left(0, \sigma_{s}^{2}\right)\right)$, theoretical Bit Error Rate $\left(B E R_{t h}\right.$ is:

$$
B E R_{t h}=\frac{1}{2} \cdot \operatorname{erfc}\left(\sqrt{\frac{E_{b}}{N_{0}+2 \alpha}}\right),
$$

with $E_{b}$ the energy per bit, $N_{0}$ the spectral density of the noise $n_{1}(t)$ and $\operatorname{erfc}$ the well known complementary error function. Fig. 4 shows the theoretical BER for different $W S R$. Performance are compared with simulation results. For the simulations, the signal $s(t)$ is an OFDM modulation with 4-QAM non-coded symbols per subcarriers. The number of subcarriers is set to 1024, producing a zero-mean Gaussian signal due to the central limit theorem [6]. The watermark $w(t)$ is also a Gaussian signal.

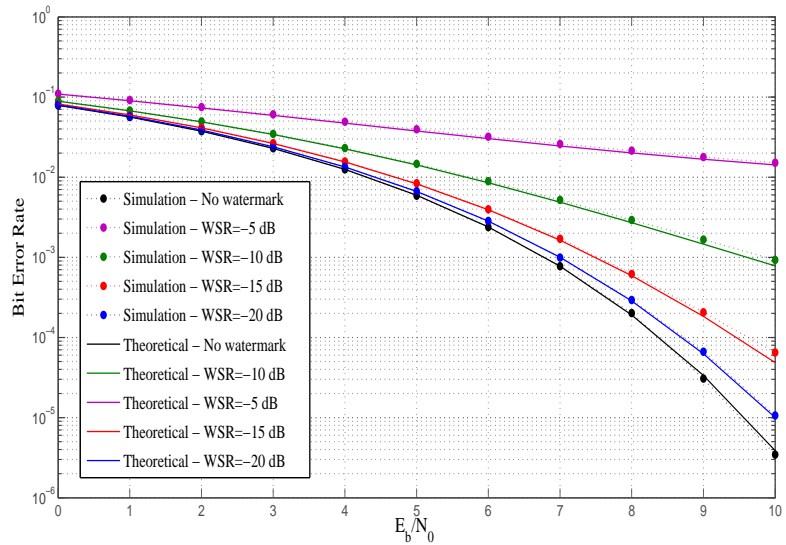

Fig. 4. Insertion sensibility: BER versus $E_{b} / N_{0}$ for several watermark power $W S R$.

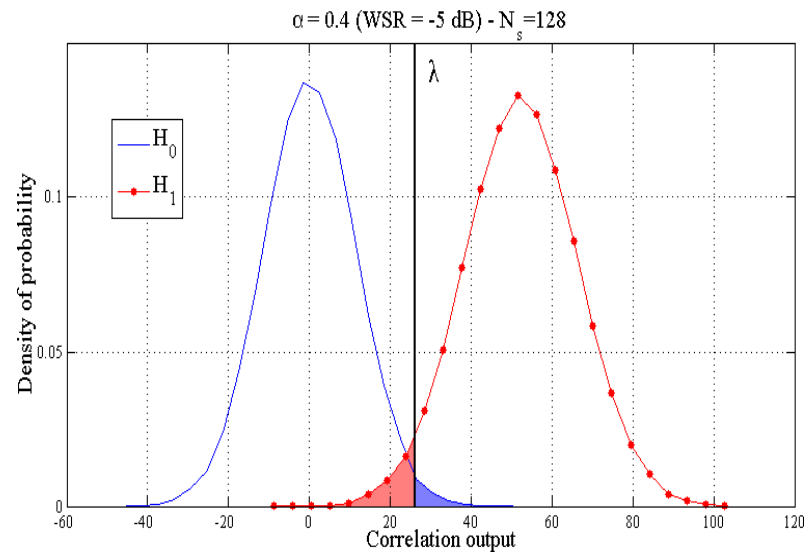

Fig. 5. Density of probability of the correlation output $u$ for $H_{0}$ and $H_{1}$.

Fig. 4 shows that the simulation performance perfectly match the theoretical curves. The results show that the watermark insertion leads to a $E_{b} / N_{0}$ degradation lower than $1 \mathrm{~dB}$ (for a target BER of $10^{-3}$ ) and for $W S R$ less than $-15 \mathrm{~dB}$.

\section{B. Signal detection characteristics}

The performance of the watermark detection are evaluated. Taking Gaussian assumptions for the signal, the code and the noise, the correlation output statistics could be derived from (4):

$$
u \sim \begin{cases}\mathcal{N}\left(0, \frac{\sigma_{n}^{2}}{N_{s}}\right) & \text { if } H_{0}, \\ \mathcal{N}\left(0, \frac{\sigma_{s}^{2}+\sigma_{n}^{2}}{N_{s}}\right)+\alpha & \text { if } H_{1} .\end{cases}
$$

Fig. 5 shows the density of probability of the correlation output $u$ for both hypothesis $H_{0}$ and $H_{1}$ (with $N_{s}=128, W S R=-5 \mathrm{~dB}$ and $S N R=-15 \mathrm{~dB}) . H_{0}$ density average is 0 while $H_{1}$ density average is $\alpha * N_{s}=51$ samples.

The probability of detection $p_{D}$ depends directly on the choice of the detection threshold $\lambda$. This choice is a tradeoff between false alarms and good detections as shown in Fig 5.

Non-detections represent the red area on Fig. 5. The non- 


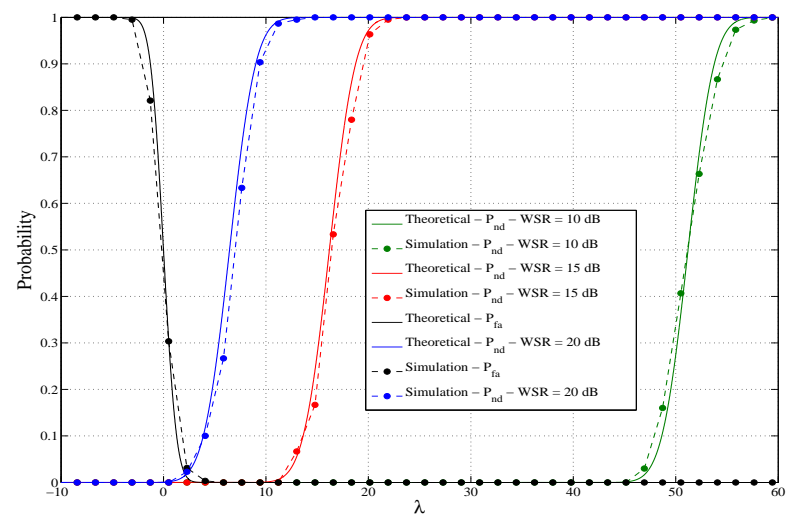

Fig. 6. False alarm probability and non-detection probability versus detection threshold $\lambda$.

detection probability $P_{n d}$ is computed by:

$P_{n d}=\operatorname{Prob}\left(c<\lambda \mid \mathcal{H}_{1}\right)=\int_{-\infty}^{\lambda} \mathcal{N}\left(0, \frac{\sigma_{s}^{2}+\sigma_{n}^{2}}{N_{s}}\right)+\alpha \mathrm{d} u$.

Thus, the theoretical non-detection probability has a function of detection threshold is given by:

$$
P_{n d}=\frac{1}{2}-\frac{1}{2} \operatorname{erfc}\left(\frac{\lambda-\alpha}{\sqrt{2} \frac{\sigma_{s}^{2}+\sigma_{n}^{2}}{N_{s}}}\right) .
$$

Finally, the detection probability $P_{d}$ is given by:

$$
P_{d}=1-P_{n d} .
$$

False alarms represent the blue area on Fig. 5. The theoretical false alarm probability is given by:

$$
P_{f a}=\operatorname{Prob}\left(c \geq \lambda \mid \mathcal{H}_{0}\right)=\frac{1}{2} \operatorname{erfc}\left(\frac{\lambda}{\sqrt{2} \frac{\sigma_{n}^{2}}{N_{s}}}\right) .
$$

Fig. 6 shows the theoretical $P_{f a}$ and the theoretical $P_{n d}$ for different $W S R$. Performance are compared with simulation results. The simulation settings are the same as for Fig. 4. The detector uses $N_{s}=8192$ samples with a code length $L=1024$. Fig. 6 shows that the simulation performance perfectly match the theoretical curves. The results show that Gaussian conditions (for the signal, the watermark and the noise) leads to an efficient detector where signals with $S N R$ down to $-20 \mathrm{~dB}$ could be detected.

\section{Simulation RESUlts}

In this section, FBMC modulation with 256 subcarriers is performed on 4-QAM non-coded symbols. It has been shown [11] that this modulation is a good candidate for the secondary use of the spectrum. Furthermore, this modulation suffers from its lack of cyclostationary features so being a good study case for the watermarking technique proposed herein. The watermarking is performed using an Hadamard code with $L=1024$.

The main feature of a detector is its sensitivity level expressed in $S N R$. In the following, the sensitivity is the minimum

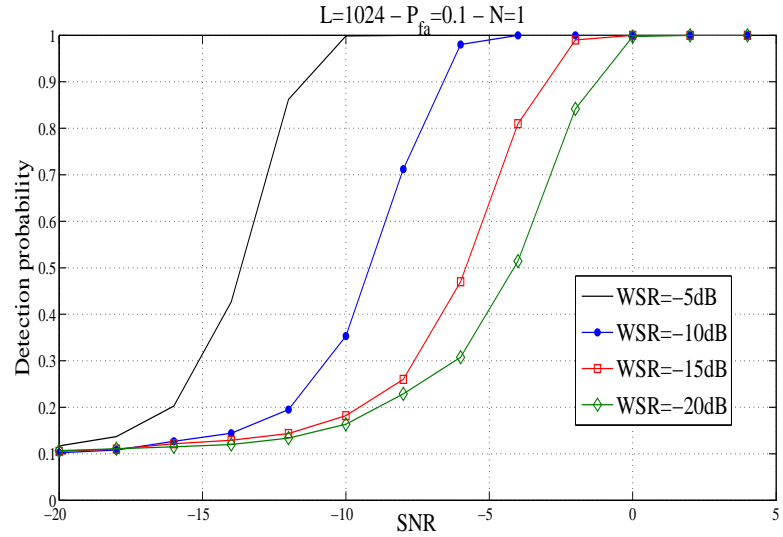

Fig. 7. Detection probability versus $S N R$ for different $W S R$.

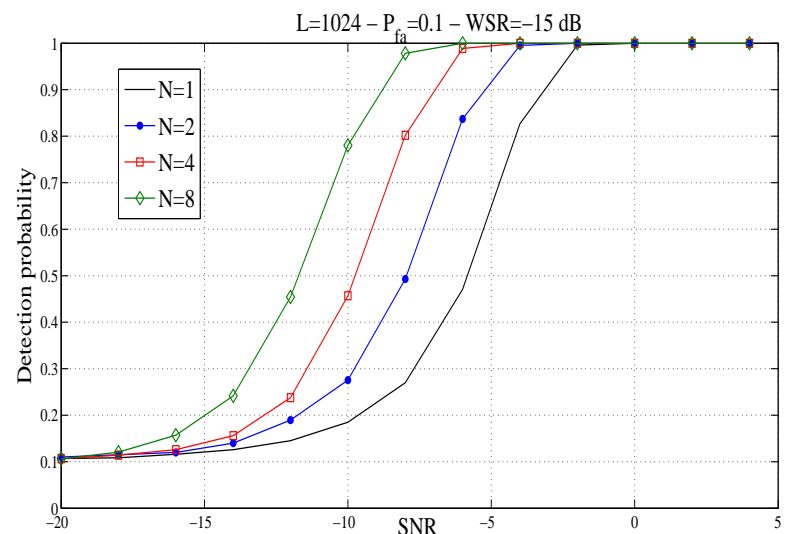

Fig. 8. Detection probability versus $S N R$ for different integration time $N$.

$S N R$ that the detector could sense with $P_{d}=95 \%$ and $P_{f a}=10 \%$.

The first results shows how increasing the detection sensitivity using 2 parameters of the systems: the insertion strength $W S R$ and the detection time $N$. Simulation results are introduced in Fig. 7 and Fig. 8. Fig. 7 shows the detection probability as a function of $S N R$ for several insertion strengths $W S R$ and for $N=1$. Fig. 8 shows the detection probability as a function of $S N R$ for a $W S R$ of $-15 \mathrm{~dB}$ and for several duration of detection $N$. In both results, the fixed threshold leads to a false alarm probability of $10 \%$.

Simulations results in Fig. 7 show that the watermark allows the detection of signals with $S N R$ between to $-1.5 \mathrm{~dB}$ and $11.5 \mathrm{~dB}$ for a $W S R$ varying between $-20 \mathrm{~dB}$ and $-5 \mathrm{~dB}$. The choice of $W S R$ depends on the targeted link quality as discussed in III-A. In the following, $W S R=-15 \mathrm{~dB}$ will be used.

Results in Fig. 8 show how improving the signal detection by increasing the integration time. Around $2 \mathrm{~dB}$ are gained by doubling the integration time leading to a detection sensitivity of $-8.2 \mathrm{~dB}$ for $N=8$.

Then, performance of the proposed detector are compared with those of the energy detector. Fig. 9 shows the sensi- 


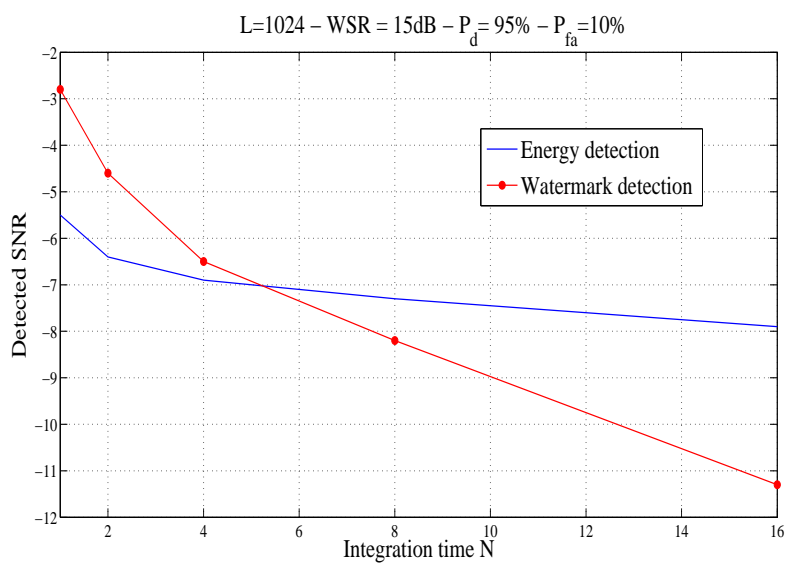

Fig. 9. Detection sensibility in SNR versus integration time $N$.
[6] R. Van Nee and R. Prasad, OFDM for Wireless Multimedia Communications, Artech House edition, 2000.

[7] P. Siohan, C. Siclet, and N. Lacaille, "Analysis and design of ofdm/oqam systems based on filterbank theory," Signal Processing, IEEE Transactions on, vol. 50, no. 5, pp. $1170-1183$, may 2002.

[8] I.J. Cox, M.L. Miller, and A.L. McKellips, "Watermarking as communications with side information," Proceedings of the IEEE, vol. 87, no. 7, pp. 1127-1141, jul 1999.

[9] Cleo Baras, Pzremyslaw Dymarski, and Nicolas Moreau, "An audio watermarking scheme based on an embedding strategy with maximized robustness to perturbations," IEEE International Conference on Acoustics, Speech and Signal Processing (ICASSP), vol. 4, pp. 357-360, May 2004.

[10] R. Kohno, R. Meidan, and L.B. Milstein, "Spread spectrum access methods for wireless communications," IEEE Communications Magazine, vol. 33, no. 1, pp. 58-67, January 1995.

[11] D. Noguet, M. Gautier, and V. Berg, "Advances in Opportunistic Radio Technologies for TVWS," EURASIP Journal on Wireless Communications and Networking, November 2011. tivity in $S N R$ as a function of the integration time $N$ for both watermark detector and energy detector. The sensitivity increases faster for the watermark detection than for the energy detection. Thus, for an integration time higher than 8 , the watermark detector outperforms the energy detector. For $N=16$, a $3.4 \mathrm{~dB}$ gain is achieved.

\section{CONCLUSIONS}

This paper proposes the initial study of a new signal detector for cognitive radio systems. It detects the watermark that has been inserted in the transmitted signal at a very low power. Theoretical performance and implementation discussion allow the setting of the system through the tradeoff between the signal detection performance and the degradation of the communication quality. In realistic conditions, simulation results show that the proposed detector outperforms the energy detection and could detect $S N R$ down to $-11 \mathrm{~dB}$ with a reasonable detection duration.

Future work is to include the proposed technique in a cooperation scheme among secondary users. All opportunistic users will be watermarked and will use this signature to allocate free spectrum in a fair and efficient way.

\section{ACKNOWLEDGMENT}

The research leading to these results was derived from the European Community's Seventh Framework Program (FP7) under Grant Agreement number 248454 (QoSMOS).

\section{REFERENCES}

[1] J. Mitola III and G. Q. Maguire Jr, "Cognitive radio: making software radios more personal," IEEE Personal Communications, vol. 6, no. 4, pp. 13-18, 1999.

[2] E. Axell, G. Leus, E.G. Larsson, and H.V. Poor, "Spectrum sensing for cognitive radio : State-of-the-art and recent advances," Signal Processing Magazine, IEEE, vol. 29, no. 3, pp. 101 -116, may 2012.

[3] H. Urkowitz, "Energy detection of unknown deterministic signals," Proceeding of the IEEE, vol. 55, no. 4, pp. 523-531, April 1967.

[4] P.D. Sutton, K.E. Nolan, and L.E. Doyle, "Cyclostationary Signatures in Practical Cognitive Radio Applications," IEEE Journal on Selected Areas in Communications, vol. 26, no. 1, pp. 13-24, jan 2008.

[5] H. Zhang, D. Le Ruyet, and M. Terre, "Signal detection for OFDM/OQAM system using cyclostationary signatures," IEEE 19th International Symposium on Personal, Indoor and Mobile Radio Communications (PIMRC 2008), sept 2008. 